\title{
WAVE DRAG REDUCTION WITH A SELF-ALIGNING AERODISK ON A MISSILE CONFIGURATION
}

\author{
C. Schnepf, O. Wysocki, and E. Schülein
}

German Aerospace Center (DLR)

10 Bunsenstr., Göttingen 37073, Germany

\begin{abstract}
A self-aligning aerodisk to reduce the wave drag on a pitching missile is numerically investigated. The motion and the Mach number were chosen to match a maneuver flight of an actual missile: pitching frequency $f$ $=7.5 \mathrm{~Hz}$, Mach number $\mathrm{M}=2.2$, and range of angle of attack $0^{\circ}$ $<\alpha<21^{\circ}$. The self-alignment was realized with a coupling of the flow solver with a $6 \mathrm{DoF}$ ( 6 degrees of freedom) tool. In the entire range of angle of attack, the drag could be reduced with the self-aligning aerodisk. A comparison with experimental data showed in parts a quite good agreement in the aerodynamic coefficients, in the shock structure, and in the alignment of the aerodisk.
\end{abstract}

\section{INTRODUCTION}

In supersonic flight, the wave drag has the major impact on the overall drag of a slender body. Hence, decreasing the wave drag while keeping a high ratio of lift over drag gives the most benefit in range and flight speed. For general aviation, this is already not easy to accomplish and even more complicated in a case of a slender body in maneuver flight achieving high angles of attack at high pitching rates. For nonlifting slender bodies of revolution, the nose has the largest impact on the wave drag. Considering a nose geometry with a limited nose length, the analytical and experimental investigations of [1] showed that an ogival geometry with a small blunt nose tip is the most beneficial to minimize the wave drag, even in comparison with a sharp cone with the same nose length. But often, these geometries have a major drawback in providing enough space to integrate avionic components. For this purpose, a simple hemispherical nose is the best choice. Unfortunately, this kind of geometry is accompanied

(C) The authors, published by EDP Sciences. This is an Open Access article distributed under the terms of the Creative Commons Attribution License 4.0 (http://creativecommons.org/licenses/by/4.0/). 


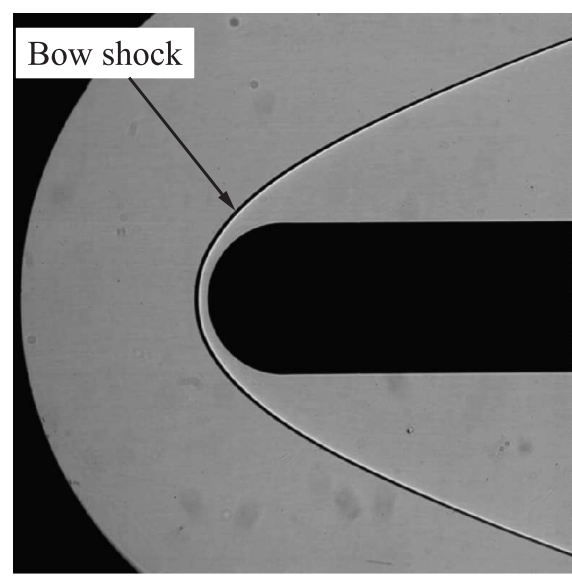

(a)

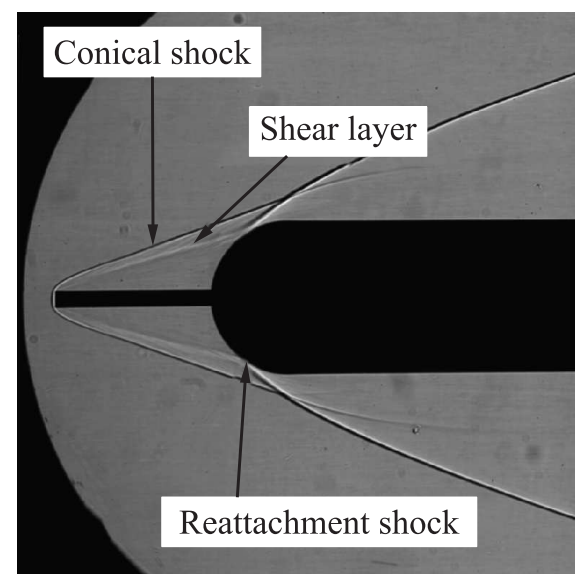

(b)

Figure 1 Shadow graphs of the bow shock without spike $(a)$ and of the spike-induced separation $(b)$ on a hemispherical model at $\mathrm{M}=5$ and $\alpha=0^{\circ}[2]$

by a strong bow shock wave in supersonic flight leading to a high wave drag (Fig. 1a).

A simple method to overcome this problem while keeping a blunt nose is the aerospike [3-5]. An aerospike is a thin rod mounted on the tip of a blunt body as shown in Fig. 1b. Slight variations on the initial design include cones, spheres, or disks that are mounted on the tip of the rod. The beneficial effect on the drag has been proven in experimental and numerical investigations since decades and has already been applied on missiles like Trident C-4 and C-5. Several review papers exist on this topic (see, e.g., $[5,6]$ ). Also, alternative concepts to the aerospike have been developed during this time. Most of them base on the same physical principle that in the case of an aerospike leads to a reduction of the wave drag. The concepts range from heating the air in front of the blunt body by laser pulses (see, e.g., [7-9]), direct-current arc discharge (see, e.g., [10]), or with a electrically heated wire pointing upstream [11]. Also, the upstream injection of a jet originating from the nose tip showed promising results. Here, jets of air (see, e.g., $[12,13]$ ) or plasma (see, e. g., [14-16]) were used. Apart from experimental investigation, also, numerical investigations were undertaken to these alternative concepts (see, e. g., [17-20]).

The physical principle of an aerospike is the formation of a separation bubble in front of a blunt nose. This bubble works for the outer supersonic flow like an actually physical body with a beneficial geometry considering the wave drag. In the ideal case, the boundary layer on the rod separates right at the tip of the rod due to the pressure rise over the small bow shock [21] that exists right in front of 


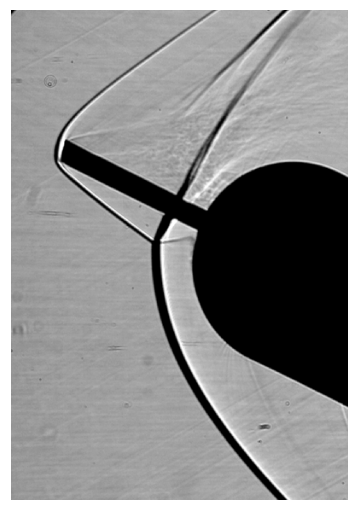

(a)

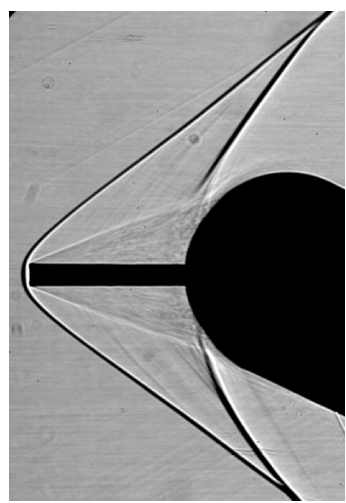

(b)

Figure 2 Shadow graphs of the flowfield for a fixed $(a)$ and an aligned spike $(b)$ at $\mathrm{M}=2$ and $\alpha=20^{\circ}[2]$

the aerospike (see Fig. 1b). The shear layer that originates from the separated boundary layer reattaches again on the blunt nose. Due to the shear layer, the outer supersonic flow shows a weaker deflection accompanied by a weaker conical shock instead of a strong bow shock (compare Fig. $1 a$ with Fig. 1b). The conical shock unites further downstream with the reattachment shock that develops due to the reattachment of the shear layer. Within the recirculation zone that is bounded by the shear layer, significantly lower pressure levels exist compared with a blunt body without an aerospike.

The efficiency of an aerospike is dependent on its length and on the Mach number. Usually, the effectiveness of a spike increases with increasing Mach number. The investigation of [2] showed that with this simple method, a drag reduction of more than $50 \%$ can be achieved as long as the spike and the blunt body show no inclination (angle of attack $\alpha$ ) to the oncoming flow. This is the major drawback of the original aerospike considering a slender body flying at high angles of attack. At an angle of attack, the original spike concepts and its derivatives fail in achieving a wave drag reduction. Even for small $\alpha$, the effectiveness decreases considerably. The beneficial effect completely disappears between $\alpha=15^{\circ}$ and $20^{\circ}[2,22]$. This is due to the transformation of the favorable symmetric shock system (see Fig. $1 b$ ) to the asymmetric one (Fig. $2 a$ ). A strong bow shock reestablishes on windward part of the hemisphere. Additionally, the bow shock interacts with the shock that originates from the spike leading to an undesired Edney type IV [23] shock-shock interaction considering heat flux.

Hence, to apply the aerospike on a maneuvering slender body, an enhancement of the original spike concept is necessary that maintains the reduction of 


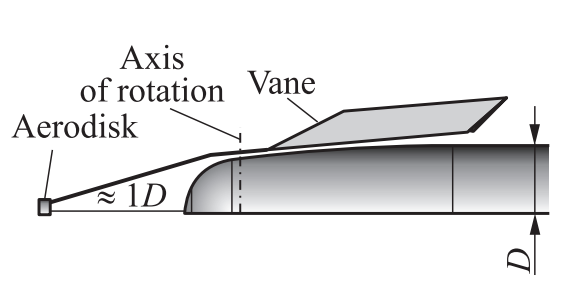

(a)

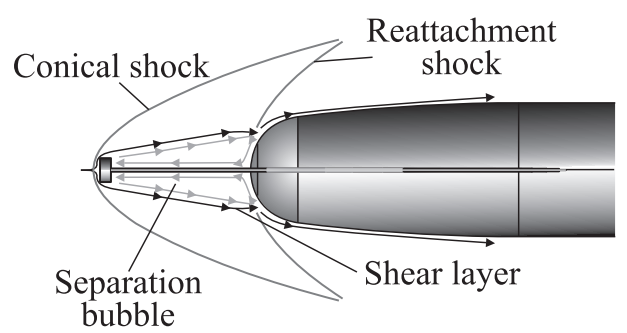

(b)

Figure 3 Model nose with self-aligning aerodisk used in the experiments of [25] and in the numerical investigation: $(a)$ top view; and $(b)$ side view

the wave drag also at high angles of attack. One method is the self-aligning aerospike concept [24]. This concept has a movable spike that can pitch about the nose of the missile. Due to that fact, the aerospike can be aligned to the oncoming flow $\alpha_{\mathrm{AD}}=0^{\circ}$ even though the main body shows an angle of attack $\alpha>0^{\circ}$. The favorable shock system in front of the nose can be sustained (Fig. 2b) and, thus, the reduction of the wave drag at the nose as it was already demonstrated in the simple "proof-of-concept experiment" of Schülein [2]. The self-aligning aerospike has been also already investigated in an advanced wind tunnel experiment of a generic missile [25].

Due to a special wind tunnel support, it was possible to investigate the selfaligning aerospike on a missile geometry pitching sinusoidal at high angular rates equivalent to the ones that occur in a realistic maneuver flight of a missile. From the aerodynamic point of view, the sinusoidal pitching motion makes the selfalignment even more ambitious. An induced flow field arises due to the motion of the missile that changes the local flow direction at the missile and at the aerospike. In the experiment of Wysocki et al. [25], the spike itself was replaced by a disk mounted on a frame. The frame itself could pitch about the nose of the missile. Due to small vanes that are also attached on this frame, the aerodisk was aligned to the oncoming flow (Fig. 3). In the following sections, this device is abbreviated with "AD." The aerodynamic forces acting on the vanes induce a pitching moment about the hinge and align the disk with the oncoming flow. With the $\mathrm{AD}$, the drag could be reduced successfully for the entire range of angle of attack $\left(0^{\circ}<\alpha<21^{\circ}\right)$ and for all Mach numbers $(1.4<\mathrm{M}<2.2)$ and frequencies $\left(f_{\max }=7.5 \mathrm{~Hz}\right)$. In accordance with the investigation of [2], the effectiveness was increasing with increasing Mach number. With the AD at the upper reversal point $\left(\alpha=21^{\circ}\right)$ of the pitching motion, still a drag reduction of $5 \%$ could be achieved $(\mathrm{M}=2.2)$.

The self-aligning aerospike concept was also investigated numerically already on a simple cylinder with a hemispherical nose at $\mathrm{M}=1.4$. The cylinder was 
pitching sinusoidal at $f=5 \mathrm{~Hz}$ and $0^{\circ}<\alpha<20^{\circ}$ [26]. A qualitative comparison with [25] showed a good agreement of the kinematic behavior of the AD. Consistent with the experiment, a perfect alignment of the $\operatorname{AD}\left(\alpha_{\mathrm{AD}}=0^{\circ}\right)$ could not be achieved over the entire pitching cycle, even though the drag was decreased also at high angles of attack.

In the present paper, the effect of a self-aligning aerodisk on a missile geometry similar to the one in [25] is investigated numerically. The body is pitching sinusoidal with $f=7.5 \mathrm{~Hz}$ at $0^{\circ}<\alpha<21^{\circ}$ and $\mathrm{M}=2.2$. This corresponds to the largest investigated pitching rate and Mach number in the experiments [25]. The main motivation of the present investigation is to make a fair comparison with the experimental data. Therefore, apart of the missile, also, the sting and the joint used in the experiments are part of the computational geometry. Like in [26], the self-alignment of the $\mathrm{AD}$ is realized with a coupling of the flow solver with a flight mechanic tool (FMT). The investigation will show if also in a numerical simulation the drag reduction will be sustained at high angle of attack on a missile geometry.

\section{COMPUTATIONAL GEOMETRY AND COMPUTATIONAL GRID}

The investigated missile geometry shown in Fig. $4 a$ has a total length $L$ of $720 \mathrm{~mm}$ and a diameter $D$ of $=36 \mathrm{~mm}$. The missile nose has a length of $72 \mathrm{~mm}$ and consists of a semispherical tip $\left(D_{\mathrm{NT}}=0.8 D\right)$ with a smooth transition to a tangent ogive. Apart of the axially symmetric body, the geometry shows four wings and rudders in X-arrangement (see black parts in Fig. $4 a)$.

The gray geometry in Fig. $4 a$ is identical with the one of the wind tunnel experiment [25] until the location of the pivot. The pivot itself is not located on the roll axis of the missile. This was necessary because of the high inertial forces in the experiment that arise due to the pitching motion, on the one hand,

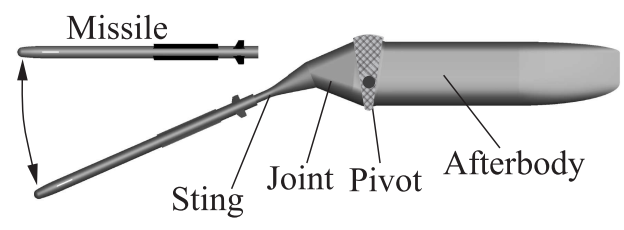

(a)

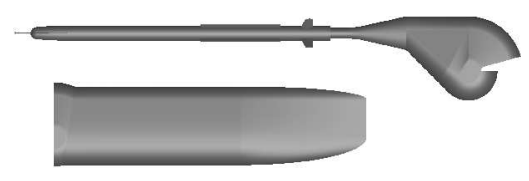

(b)

Figure 4 Computational geometry: (a) superimposed geometry; and (b) separated geometry 
and the least possible interference of the special wind tunnel support with the aerodynamics of the missile, on the other hand.

Considering a fair comparison of the experimental data with the one of the numerical simulation, it is sufficient to have identical missile and sting geometries. The geometry downstream of the sting should have no impact on the overall missile aerodynamics since this investigation is dealing with the supersonic flow.

Actually, the computational geometry shown in Fig. $4 a$ consists of two parts shown in Fig. $4 b$ which were separately meshed and later superimposed. To get a proper computational grid, the hole-cutting Chimera technique [27] was applied in the simulations. This was necessary to realize the motion of the joint. In the area marked by squares, an interpolation of the flow properties between both grids at the surface takes place (see Fig. $4 b$ ). Since only a pitching motion in the investigation is considered, it is sufficient to use a half model instead of full three-dimensional (3D) configuration in order to save computational time.

To simulate the self-alignment of the AD, the third geometry, respectively grid, is necessary that contains the $\mathrm{AD}$ geometry. In the experiments, the aerodisk consists of a porous material with a diameter of $D_{\mathrm{AD}} \approx 0.14 D$ and a distance to the nose of about $2 D$. In the numerical investigation, the aerodisk was simulated as a viscous wall. In this specific case, a proper boundary condition to take the effect of the porosity into account was not available in the flow solver. Besides the material of the aerodisk, the AD of the numerical investigation is identical with the one of the experiment (see Fig. 3). Like for the rotationally motion of the joint, the pitching motion of the $\mathrm{AD}$ about the nose of the missile is realized with the hole-cutting Chimera technique.

The computational grids were generated with Centaur ${ }^{\mathrm{TM}}$. For the missile geometry, mainly, a structured approach was chosen to discretize the surface of the missile, the boundary layer, and the vicinity on lee side of the missile. Only at challenging positions like at the junction of the wings and rudders with the cylindrical body, a locally hybrid mesh was created consisting of prismatic elements and tetrahedrons. The surface of the half model was discretized with 98 cells in circumferential direction with a maximum length in roll axis direction of $0.002 \mathrm{~L}$. In Fig. 5, the light gray zones indicate where a structured grid approach was used. In front of the nose tip, the missile grid shows also a small grid spacing to achieve a good resolution of the aerodisk induced flow structure (see Fig. $6 e$ ). The superimposed Chimera grid without the grid of the AD consists of $12 \cdot 10^{6}$ grid nodes. A more detailed description of this Chimera grid approach is given in [28].

The geometry with the cyan edges in Fig. $6 a$ illustrates the dimension of the AD-grid. For the AD itself, a hybrid mesh approach was chosen to discretize the surface and the boundary layer region. But at the bottom of the AD-grid, two layers of structured hexahedron blocks (1SL/2SL) are included (see Fig. $6 b$ ). In total, this grid has $2.2 \cdot 10^{6}$ grid nodes. 


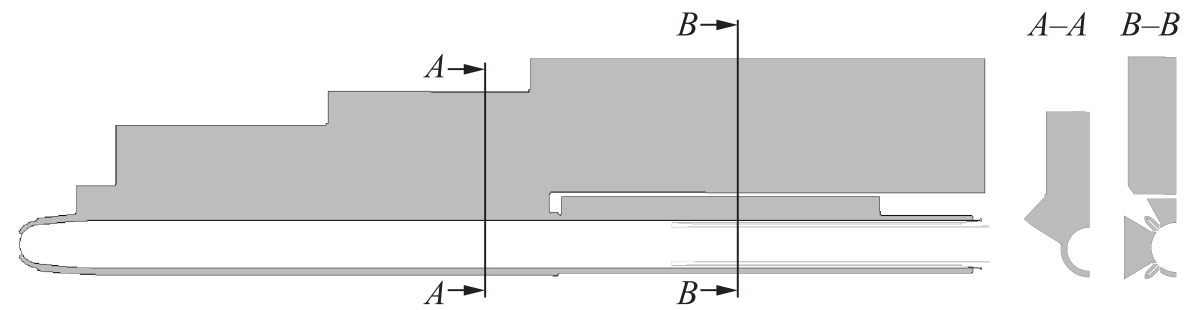

Figure 5 Missile grid approach. At the light gray zones, the mesh shows a structured grid approach

The structured layer at the bottom (1SL) of the AD-grid has larger cells than the second (2SL) (see Fig. 6b). This is done to ensure more or less at all $\alpha_{\mathrm{AD}}$ a similar cell sizing of the $\mathrm{AD}$ grid and the missile grid at the borders where the Chimera interpolation takes place. To ensure this was the most ambitious part of this Chimera grid topology. Similar cell sizing is a key requirement to minimize errors due to the Chimera interpolation.

The layer 1SL is used for an interpolation between larger cells that takes place outside of the boundary layer of the missile (see Fig. $6 f$ ). The frayed cyan line shows the interpolation zone where the flow properties are interpolated from the missile grid to the $\mathrm{AD}$ grid. At the green frayed line, it is the other way around.

If the $\mathrm{AD}$ is close to the boundary layer of the missile, the Chimera interpolation takes place mainly between the 2SL layer of the AD-grid and the missile grid (see Fig. 6d). A so-called hole definition determines if the interpolation takes place at the first or at the second structured layer. The hole is rigidly connected to the missile grid. It is shown in Fig. $6 a$ in red together with the surface of the missile in orange and the AD-grid. All geometries are in right arrangement for the case $\alpha_{\mathrm{AD}}=0^{\circ}$. Within the hole definition, unwanted nodes of the computational grid are cut out to get a reasonable Chimera grid.

The effect of the hole cutting is illustrated in Figs. $6 c$ and $6 d$. For the cutout of the nodes within the AD geometry, the second hole is necessary (not shown). A further description of the Chimera grid approach to realize the motion of the AD relatively to the missile can be found in [26]. This work also includes a validation of the $\mathrm{AD}$ Chimera grid.

\section{NUMERICAL APPROACH}

In this investigation, the unsteady 3D time accurate Reynolds averaged NavierStokes equations (URANS) have been solved with the finite volume flow solver 


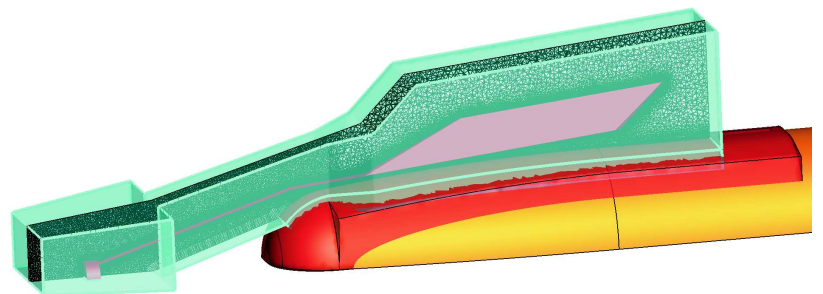

(a)

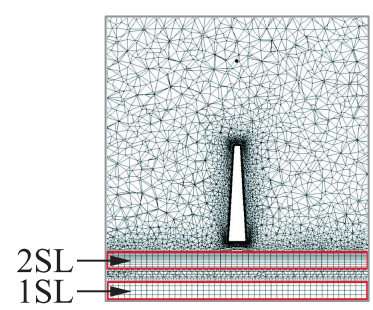

(b)

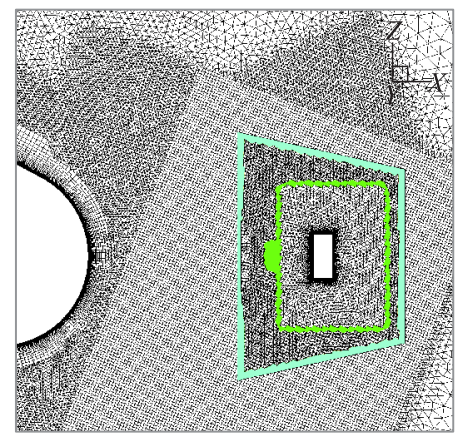

(e)

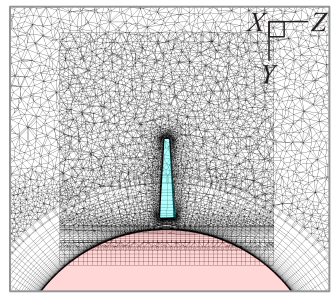

(c)

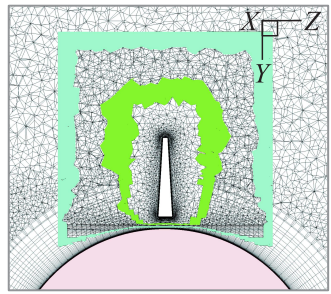

(d)

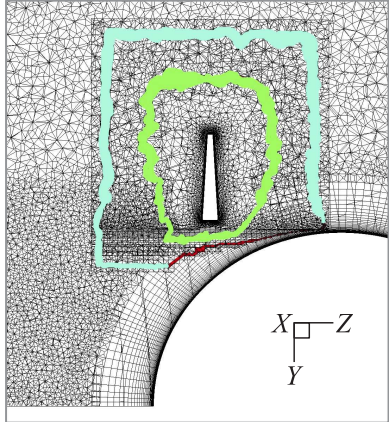

(f)

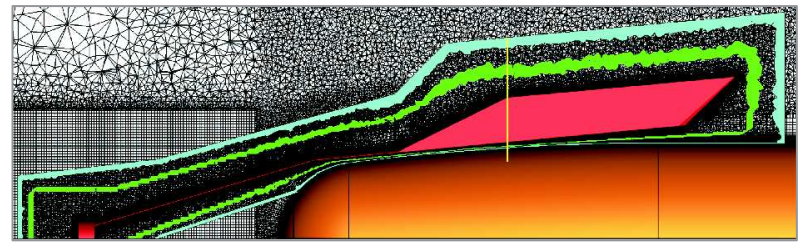

(g)

Figure 6 The AD Chimera grid: (a) longitudinal cut of the AD grid and outer boundary of the $\mathrm{AD} ;(b) \mathrm{AD}$ cross section; $(c)$ and $(d)$ cross sections of the computational grid without $(c)$ and with $(d)$ hole cutting for $\alpha_{\mathrm{AD}}=0^{\circ}$ (for cut position, see yellow line in Fig. $6 g)$; (e) symmetry plane of the computational grid with Chimera interpolation zones; $(f)$ cross section of the computational grid for $\alpha_{\mathrm{AD}} \neq 0^{\circ}$; and $(g)$ longitudinal cut of the computational grid with interpolation zones 
TAU-code [29]. The flow properties of the numerical simulation are: $\mathrm{M}=2.2$, $\operatorname{Re}=296000, T_{0}=315 \mathrm{~K}$, and $u_{\infty}=782.7 \mathrm{~m} / \mathrm{s}$. Except for the aerodisk front, the boundary layer on the $\mathrm{AD}$ and on the missile is assumed to be turbulent. This simplification is necessary since no information from the experiment exists where transition takes place. Preliminary studies with a $\gamma-\operatorname{Re}_{\theta t}$ transition model showed no reasonable results in detecting the transition of the shear layer in front of the nose.

A TAU specific adapted version of the $k-\omega$ turbulence model [30] was used in the present investigation to model the turbulent boundary and shear layers. Additionally to the standard formulation, it uses the same shear-stress correction as proposed by the shear stress transport model from Menter [31]. In the investigation of [32], a standard $k-\omega$ model showed already good agreements with experimental data for a NASA dual Control Missile at $\mathrm{M}=1.75$ in the range of $0^{\circ}<\alpha<24^{\circ}$. To know how the $k-\omega$ model copes with the aerodisk induced flow field, an additional study of different turbulent models (sequential approximate optimization (SAO), $k-\omega, k-\omega$ shear stress transport (SST), explicit algebraic Reynolds-stress model (EARSM)) at this region was undertaken. Between the two-equation turbulence models, no significant discrepancy occurred for the tested cases at $\mathrm{M}=2.2$. The flow structure and $C_{D}$ have been quite similar (maximum deviation $<5 \%$ ). But at $\mathrm{M}=1.4$ with the $k-\omega \mathrm{SST}$, a retarted separation [5] occurred. Here, the $k-\omega$ models showed better results in comparison with experimental data.

For flux discretization, a second-order AUSMDV upwind scheme is used with a least square gradient reconstruction. The discretization in time is done by an explicit Runge-Kutta scheme. The physical time step of the simulation is $\Delta t=50 \mu$ s corresponding to 2667 physical time steps per period. The sinusoidal pitching motion of the missile has a frequency of $7.5 \mathrm{~Hz}$. A pitching cycle starts with an upstroke from $\alpha=0^{\circ}$ to $21^{\circ}$ followed by a down stroke back to $\alpha=0^{\circ}$. With this motion definition the maximum change in $\alpha$ per physical time step is quite small: $\Delta \alpha=0.025^{\circ}$. With the velocity of the oncoming flow of $u_{\infty}$ and with the diameter of the missile $D$, the reduced frequency of the motion is $k_{D}=0.001$.

The self-alignment of the AD is achieved due to a coupling of the flow solver with an FMT. The FMT uses the aerodynamic forces and the inertia tensor for input values and calculates the relative motion of the $\mathrm{AD}$ with respect to the missile (Output: $\alpha_{\mathrm{AD}}$ and pitch rate $\omega_{\mathrm{AD}}$ ). Due to the bearing, the only degree of freedom for the $\mathrm{AD}$ is a pitching motion about the axis of rotation (see Fig. 3a). For simplicity in the numerical simulation, the center of mass is located on the pitching axis of the $\mathrm{AD}$, while in the experiment, the center of mass is some distance downstream of the pivot. The FMT and the flow solver are coupled several times per physical time step. The amount of coupling cycles was defined to be 4 times in the present case. Before each interchange of the input and output data, 25-50 inner iterations were done on solver side and 5 inner 
iterations on the flight mechanic side. A more detailed description of the FMT implemented in TAU can be found in [33]. In general, it uses the approach of $[34]$.

\section{RESULTS AND DISCUSSION}

In this section, the results of the numerical investigation of a self-aligning aerodisk attached to a missile in pitching motion is presented. A comparison is made with the data of an equivalent experimental investigation [25]. The experimental data is the outcome of an averaging of several pitching cycles. The aerodynamic forces have been measured with a strain gauge balance and for each pitching cycles, a correction of the forces was applied. This correction was necessary due to the large inertial forces that develop on the pitching wind tunnel model.

In Fig. $7 a, C_{D}$ vs. $\alpha$ is shown for the missile with $\mathrm{AD}$ for the pitching maneuver and for static angles of attack for the experiment and for the numerical simulation. The starting point of the dynamic simulation was a converged solution at $\alpha=0^{\circ}$ and the aerodisk was perfectly aligned to the oncoming flow $\left(\alpha_{\mathrm{AD}}=0^{\circ}\right)$. At the end of the first simulated period, the dynamic maneuver simulation was already converged. A partially simulated third period with a smaller physical time step $(\Delta t / 2)$ showed no significant differences. In Fig. $7 a$, only the result of the second period is shown. The numerically determined trend of $C_{D}$ has a hysteresis-like shape in counterclockwise direction. This trend of the hysteresis is mainly due to the induced angle of attack. But also, the misalignment

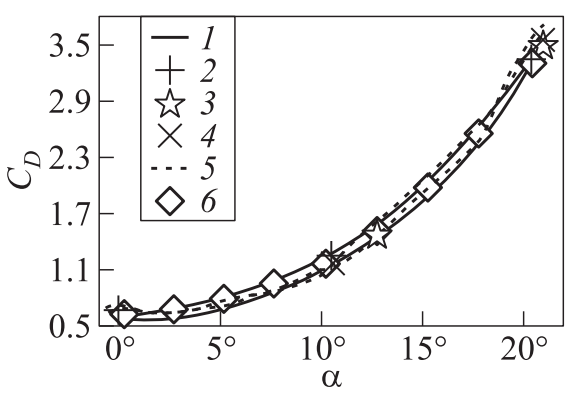

(a)

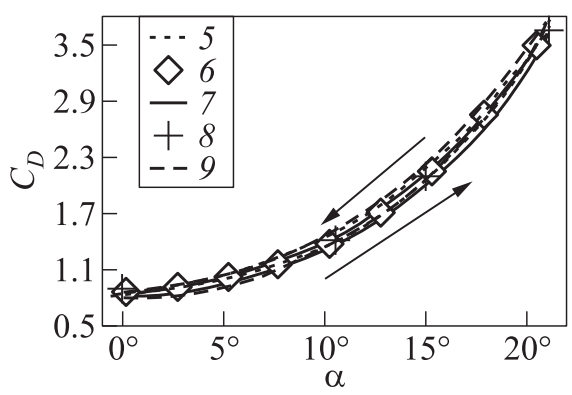

(b)

Figure 7 Drag coefficients of the missile with $(a)$ and without $(b)$ self-aligning aerodisk: $1-2$ nd period CFD; $2-$ CFD static 6 DoF; $3-$ CFD static $\alpha_{\mathrm{AD}}=0^{\circ}$; 4 - CFD static $\alpha_{\mathrm{AD}}=7.5 ; 5$ - experiments, dynamic; 6 - experiments, static; 7 CFD dynamic; 8 - CFD static; and $9-$ CFD dynamic with AD offset 
of the $\mathrm{AD}$ is responsible for that, like it will be shown later. Aside from the reversal point of the motion, the data of the dynamic numerical simulation show only small deviations from the experimental data. The slope of the experimental curve for $\alpha<8^{\circ}$ seems to be smaller than for the one of the simulation. At higher angle of attack, it is the other way around. It seems that on the base shape of the experimental curve, a wavy shape is superimposed. The reason for that and for the large differences at the reversal points between the experiment and the numerical simulation could be the fact that in the experiment, the center of mass of the $\mathrm{AD}$ is not located on the axis of rotation of the AD. At the reversal points, the largest angular acceleration exists. This fact combined with the location of the center of mass leads to additional pitching moments acting on the $\mathrm{AD}$ in the experiment. A misalignment due to this additional pitching moment is most likely, which influences also the drag coefficient of the missile. Due to the large angular acceleration, large inertial forces arise also at the missile that can outnumber the aerodynamic forces. The applied correction to the strain gauge data could be a possible source of an error. The inertial forces also cause a bending of the sting and, hence, a change in the actual angle of attack. This, of course, was also considered in the processing of the experimental data but remains a possible source of an error. The bending of the sting is the reason why for the experiment, also, the negative angle of attack data exists.

For the static numerical data 2, the alignment of the $\mathrm{AD}$ was also determined with a coupling of the flow solver with the FMT. A comparison of these data with the static experimental data shows a good agreement. In case of symbols 3 , the $\mathrm{AD}$ has a perfect alignment to the oncoming flow $\left(\alpha_{\mathrm{AD}}=0^{\circ}\right)$. Here, the alignment was defined in the flow solver. This leads to lower $C_{D^{-}}$-values in comparison with the data marked by symbols 2 . The symbols 4 at $\alpha=21^{\circ}$ show the result for an alignment of $\alpha_{\mathrm{AD}}=7.5^{\circ}$. In contrast to the perfect alignment, this alignment leads to an increase in $C_{D}$ in comparison with symbols 2 . Such large misalignments did not occur in the numerical simulations but in the experiments of the maneuvering missile. Considering only the drag at the hemispherical nose tip shown in Tables 1 and 2, the effect of a misalignment on $C_{D}$ is even more evident. Comparing rows 1, 2, and 3 in Table 1, it is obvious that with increasing misalignment, the drag increases. But even with the large misalignment, a drag reduction can be achieved in comparison with the case without AD. Comparing the different cases (perfect alignment/FMT-alignment) in Tables 1 and 2 separately, it is found that the drag at the hemisphere is similar even though the difference in angle of attack is quite large.

In Fig. $7 b$, the drag coefficient vs. $\alpha$ is shown for the experiment and the numerical simulation for the missile without AD. Like for the simulation with $\mathrm{AD}$, a hysteresis occurs showing the same direction. But the width of the hysteresis seems to be narrower like for the case with AD. This is due to the fact that the induced angle of attack is just responsible for trend of $C_{D}$. Like for 
Table 1 Drag coefficient at the hemisphere and nose for $\alpha=21^{\circ}$

\begin{tabular}{llcc}
\hline & \multirow{2}{*}{ Case } & \multicolumn{2}{c}{$C_{D}$} \\
\cline { 3 - 4 } & & Hemisphere & Nose (hemisphere + ogive) \\
\hline 1 & Static, $\alpha_{\mathrm{AD}}=0^{\circ}$ (ideal) & 0.1353 & 0.4413 \\
2 & Static, $\alpha_{\mathrm{AD}} \approx 3.1^{\circ}$ & 0.1863 & 0.4756 \\
3 & Static, $\alpha_{\mathrm{AD}}=7.5^{\circ}$ & 0.324 & 0.6194 \\
4 & Dynamic, $\alpha_{\mathrm{AD}} \approx 3^{\circ}$ & 0.1860 & 0.4754 \\
5 & Static, without $\mathrm{AD}$ & 0.520 & 0.803 \\
\hline
\end{tabular}

Table 2 Drag coefficient at the hemisphere and nose for $\alpha=10.5^{\circ}$

\begin{tabular}{llcc}
\hline & \multirow{2}{*}{ Case } & \multicolumn{2}{c}{$C_{D}$} \\
\cline { 3 - 4 } & & Hemisphere & Nose (hemisphere + ogive) \\
\hline 1 & Static, $\alpha_{\mathrm{AD}}=0^{\circ}$ (ideal) & 0.137 & 0.226 \\
2 & Static, $\alpha_{\mathrm{AD}} \approx 2.9^{\circ}$ & 0.189 & 0.274 \\
3 & Static, without AD & 0.5233 & 0.59 \\
\hline
\end{tabular}

the case with $\mathrm{AD}$, also for the case without $\mathrm{AD}$, the comparison of $C_{D}$ between the experimental and numerical data shows a good agreement for the static and dynamic data. This time, the agreement is even better and applies also at the reversal points. Also, here, the missing effect of the misalignment of the $\mathrm{AD}$ could be the reason for the better agreement. A difference exists in the width of the hysteresis. Here, the experiment shows a narrower hysteresis.

For the numerical simulation in Fig. $7 b$, the drag coefficient is plotted also for the case with $\mathrm{AD}$. A steady offset $\Delta C_{D}$ is added to the $\mathrm{AD}$ data to make a comparison with the case without AD more easy. Due to the correction, both dynamic simulations (without and with $\mathrm{AD}$ ) show at $\alpha=0^{\circ}$ the same $C_{D}$. Considering the data of the numerical simulation, it can be seen that in case without $\mathrm{AD}$, the drag shows an increase in drag from the beginning (upstroke and downstroke), while for the simulation with $\mathrm{AD}$, only for the down stroke a similar behavior occurs. For the upstroke for $\alpha<2.5^{\circ}$, a kind of a plateau value exists. This different behavior for the downstroke and the upstroke has to do with the misalignments. The downstroke shows in general much larger misalignment leading to an open asymmetric seperation and an adverse pressure distribution at the hemisphere (Fig. 8). This issue will be discussed in more detail at a later point of this study.

In Fig. 9, the misalignment that occurs in the numerical simulation is presented for different pitching cycles. The fundamental trend of the curve of misalignment is identical for the two first and the partially simulated third period. 


\section{\begin{tabular}{c|c|c|c|c|c|}
\multicolumn{7}{c}{$c_{p}$} \\
\multicolumn{1}{c|}{$|c|$} & & & \\
\hline-0.05 & 0.06 & 0.17 & 0.28 & 0.39 & 0.50
\end{tabular}}

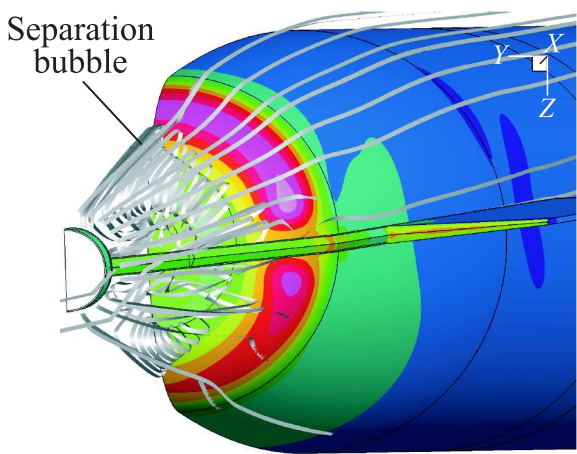

(a)

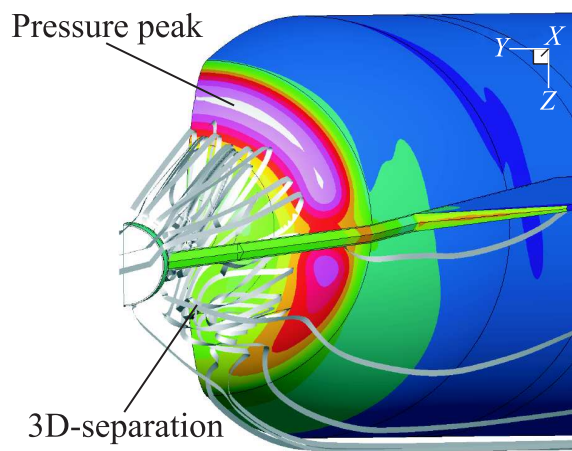

(b)

Figure 8 Difference in separation at $\alpha \approx 2^{\circ}$ between the upstroke $(a)$ and downstroke $(b)$ due to different $\alpha_{\mathrm{AD}}:(a) \alpha_{\mathrm{AD}}=0^{\circ}$; and $(b) \alpha_{\mathrm{AD}} \approx-1.8^{\circ}$

Only the harmonic component shows somehow a small phase shift. Generally, with increasing $\alpha$, the misalignment increases reaching a local minimum for the pitch up cycle at $\alpha=21^{\circ}$. For the first part of the downstroke, the misalignment increases further reaching a global minimum before it decreases again. In general, the downstroke shows larger misalignments than the upstroke. The trend

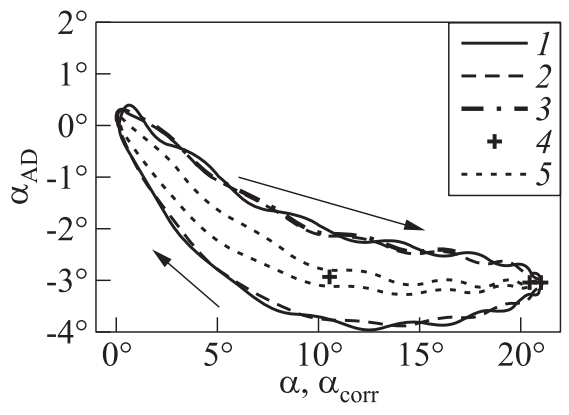

Figure 9 Misalignment of the AD: 1 1st period; $2-2$ nd period; $3-3$ rd period; $4-$ static $\alpha$; and $5-2$ nd period corrected $\left(\alpha_{\mathrm{ADcorr}}=\alpha_{\mathrm{AD}}+\alpha_{i}\right)$

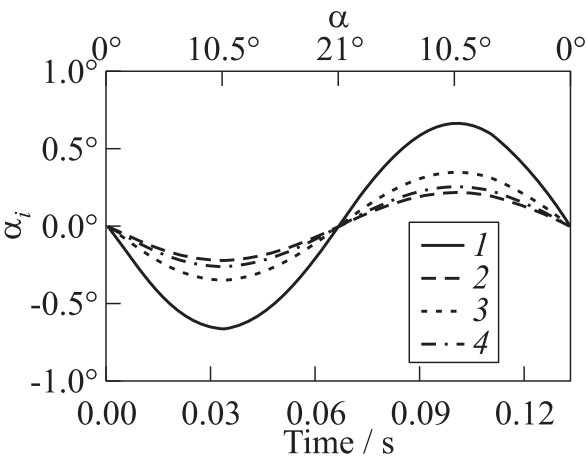

Figure 10 Induced angle of attack vs. the periodic time: $1-\alpha_{i, \text { nosetip }} ; 2-$ $\alpha_{i, \text { rear }} ; 3-\alpha_{i, \text { wing }} ;$ and $4-\alpha_{i, \text { rudder }}$ 
of the misalignments in Fig. 9 is identical with the curve of misalignment shown in [26]. But for the pitching motion with $f=5 \mathrm{~Hz}$ at $\mathrm{M}=1.4$, slightly larger misalignments at the upper reversal point and for the global minimum occur $\left(3.6^{\circ}\right.$ and $\left.4.5^{\circ}\right)$.

The misalignments for the static angle of attack are right in the middle of the hysteresis of the dynamic misalignments. At $\alpha=21^{\circ}$, the static value fits quite well with the dynamic value. An enduring nonlinear effect due to the fast pitching motion on the alignment of the $\mathrm{AD}$ seems to be not significant at $\alpha=21^{\circ}$ in the numerical simulation. In general, the pitching motion does not have a large effect on the alignment of the AD. This is indicated by the curve 5 in Fig. 9. For this curve, a correction for $\alpha_{\mathrm{AD}}$ is applied: $\alpha_{\mathrm{ADcorr}}=\alpha_{\mathrm{AD}}+\alpha_{i}$ with $\alpha_{i}$ being the induced angle of attack due to the motion at the position of the nose tip. With this simple correction, the hysteresis gets more narrow and the curves approach the static values. In [28], a detailed description is given how the effective angle of attack is changing along the missile on the specific geometry and pivot that is investigated in this work. Figure 10 shows the order of the induced angle of attack for one periodic time for several positions at the missile.

Schnepf et al. [28] and Wysocki et al. [25] have written that the misalignment occurs due to the fact that at the vanes, the local flow direction $\alpha_{O}$ is influenced by the missile. Of course, this is true but with increasing misalignment, also the drag and the lift that develop on the aerodisk are responsible for the behavior of the AD. For example, with increasing misalignment (negative $\alpha_{\mathrm{AD}}$ ), the moment arm for the drag at the aerodisk increases leading to a moment that would increase the misalignment. The sketch in Fig. 11 illustrates that not just the local flow direction at the vanes is responsible for the alignment of the $\mathrm{AD}$.

Figure 12 presents a comparison of the experimental misalignments with the ones of the numerical simulation. In contrast to the numerical simulation, the curve of misalignment of the dynamic experiment shows large amplitudes. As discussed above, these amplitudes are assigned to the fact that in the experiments, the center of mass is not located on the pitching axis of the AD. But the general trend of the experimental curve is similar to the one of the CFD. A strong increase in misalignment occurs for the lower angle of attack $\left(\alpha<10^{\circ}\right)$ followed by a flattening out of the curve approaching $\alpha=21^{\circ}$. This flattening out occurs also for the numerical simulation. One possible reason could be the fact that with increasing $\alpha$, the vane of the $\mathrm{AD}$ moves away from the regions where a large difference between the local flow direction and the direction of the oncoming flow exists (Fig. 13).

At very high angle of attack, the vane even interacts with the wake flow of the separated boundary layer originating from the missile and not with the attached boundary layer. The fact that with a misalignment the lateral and vertical distance between the vane and the missile increases could also play a role 


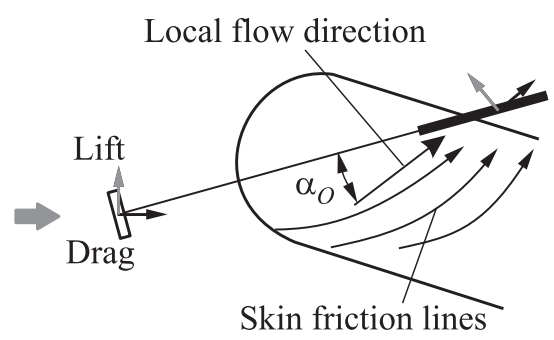

Figure 11 Sketch of the forces and moments that act on the $\mathrm{AD}$

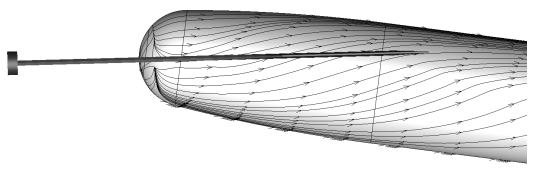

(a)

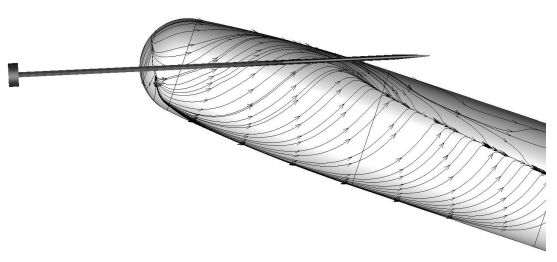

(c)

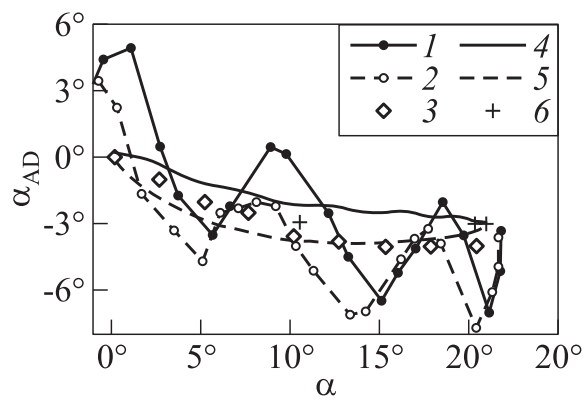

Figure 12 Comparison of the experimental (1-3) and numerical (4-6) misalignments: 1 - dynamic up; $2-$ dynamic down; 3 - static; 4 - period up; 5 - period down; and 6 - static $\alpha$ CFD

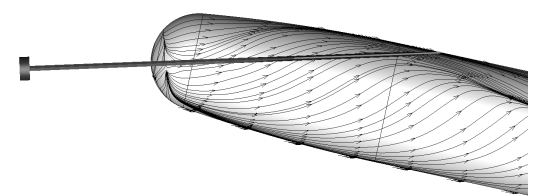

(b)

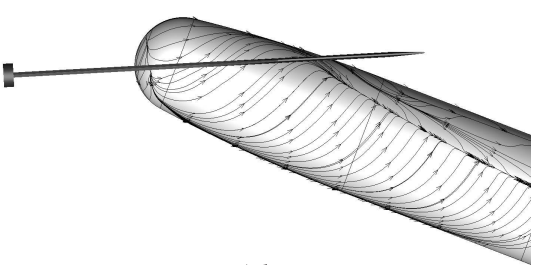

(d)

Figure 13 Skin friction line pattern at the missile close to the region of the AD: (a) $\alpha=7^{\circ} ;(b) 12^{\circ} ;(c) 18^{\circ}$; and $(d) \alpha=21^{\circ}$

(Fig. 14) because with increasing distance, the influence of the flow on the vane that itself is influenced by the missile decreases.

The ability to reduce the wave drag also at high angle of attack is the intended purpose of the self-aligning aerodisk. Figure 15 shows the percentage reduction of the drag due to $\mathrm{AD}$ in comparison to the drag of a missile without aerodisk. As expected, the efficiency decreases with increasing angle of attack considering the curve for the numerical simulation. But still at $\alpha=21^{\circ}$, a re- 


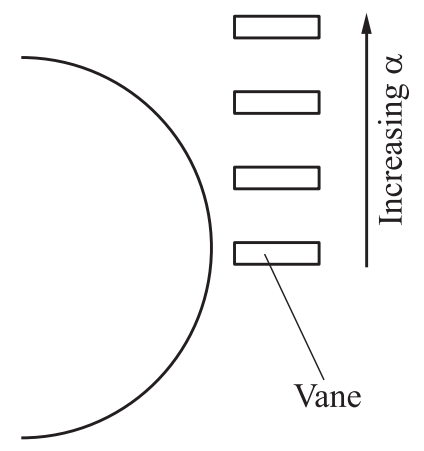

Figure 14 Sketch of the motion of the vane relative to the missile in a cross section with increasing $\alpha$ duction of about $5 \%$ remains. The decrease in efficiency has nothing to do with the misalignment rather the impact of the drag reduction at the nose on the overall drag. Figure 16 shows the share of the drag of the hemispherical nose on the total drag for the missile with and without AD. Above $\alpha \approx 5^{\circ}$, the share drops from plateau value of about $25 \%$ to about $5 \%$ at $\alpha=21^{\circ}$ for the case with $\mathrm{AD}$. Also, for the case without aerodisk, the share of the hemispherical nose on the total drag is just about $15 \%$ at $\alpha=21^{\circ}$, even though on the blunt nose, a huge wave drag exists. Hence, a reduction of the wave drag at the nose at high angles of attack does not have that impact anymore on the overall drag.

The difference in drag reduction between the upstroke and the downstroke in case of the numerical simulation can be assigned to the misalignment. Remarkable is that the maximum in drag reduction occurs at $\alpha \approx 2.5^{\circ}$ and not at $\alpha \approx 0^{\circ}$ (numerical simulation). In this $\alpha$ range, the impact of the drag of the hemisphere on the overall drag is constant for the case with AD. Combination with the delayed onset of the increase in drag for the case with $\mathrm{AD}$ for small $\alpha$ in comparison with the case without $\mathrm{AD}$ (see Fig. $7 b$ ) leads to the maximum in drag reduction at $\alpha \approx 2.5^{\circ}$.

A comparison of the drag reduction between the numerical investigation and the experiment shows the impact of the discrepancy between both data sets at

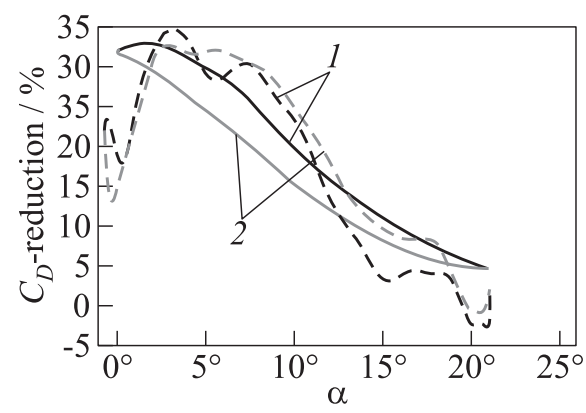

Figure 15 Numerical (solid curves) and experimental (dashed curves) $C_{D^{-}}$ reduction: 1 - up; and $2-$ down

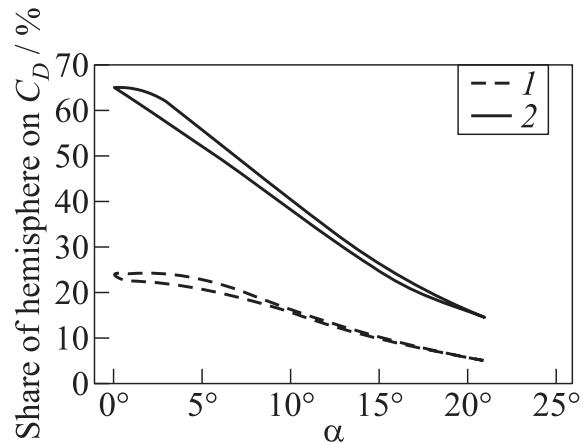

Figure 16 Numerically determined share of the hemisphere on the total drag with (1) and without (2) AD 
the reversal point for the case with $\mathrm{AD}$. Also, the fact that in the experiment, the data for the case with $\mathrm{AD}$ show a smaller slope for small angle of attack in comparison with the simulation can be seen in Fig. 16 and explains the larger maximum in drag reduction for the experiment (see Fig. 15). But in total the drag reduction has the same order of magnitude.

Figure 17 has the intension to make a comparison between the shock structure of the numerical simulation with the shock structure that occurs in the experiment. The right column shows schlieren photographs from the experiment. With red lines, the shock structure is retraced. These lines were extracted and superimposed to the density gradient in the pitching plane (CFD) shown in the left

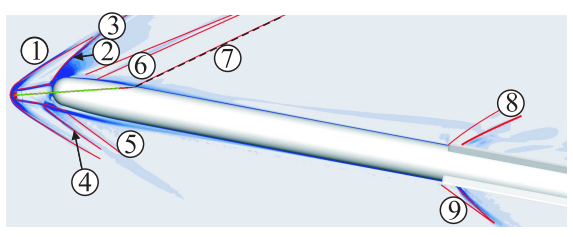

(a)

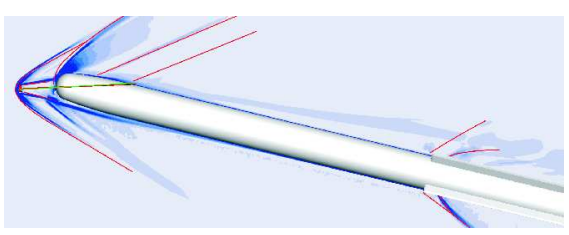

(c)

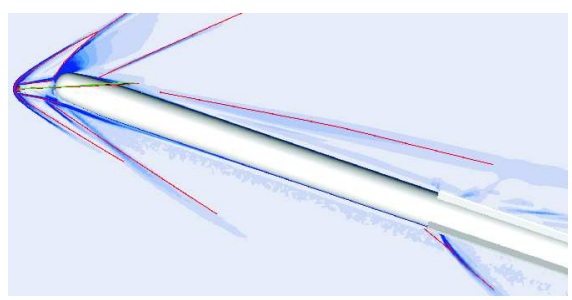

(e)

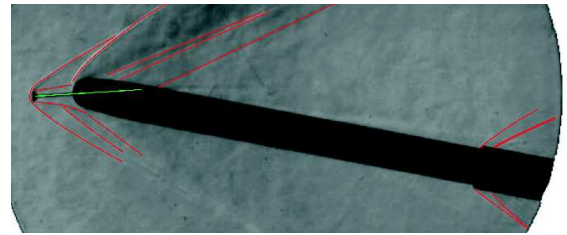

(b)

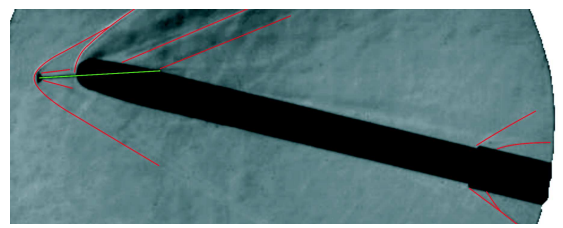

$(d)$

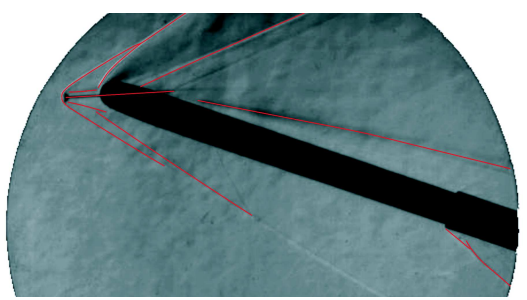

$(f)$

Figure 17 Comparison of the shock structure on the pitching missile with AD between the experiment and the numerical simulation: left column - CFD + shock structure; right column - schlieren photographs + shock structure; $(a) \alpha=10.5^{\circ}$ downstroke, $\alpha_{\mathrm{AD}, \mathrm{CFD}} \approx 3.8^{\circ} ;(b) \alpha \approx 10.5^{\circ}$ downstroke, $\alpha_{\mathrm{AD}, \mathrm{CFD}} \approx 3.5^{\circ} ;(c) \alpha=13.4^{\circ}$ upstroke, $\alpha_{\mathrm{AD}, \mathrm{CFD}} \approx 2.4^{\circ} ;(d) \alpha=13.4^{\circ}$ upstroke, $\alpha_{\mathrm{AD}, \mathrm{CFD}} \approx 3.4^{\circ} ;(e) \alpha=18.6^{\circ}$ upstroke, $\alpha_{\mathrm{AD}, \mathrm{CFD}} \approx 2.7^{\circ} ;$ and $(f) \alpha=18.6^{\circ}$ upstroke, $\alpha_{\mathrm{AD}, \mathrm{CFD}} \approx 3.32^{\circ}$ 


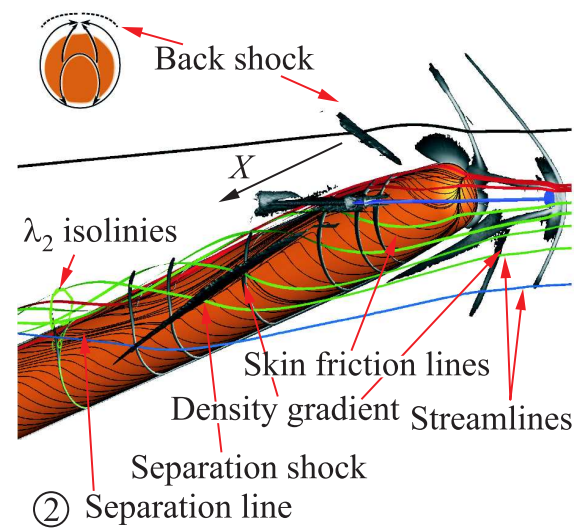

Figure 18 Sketch of the shock and flow structure and skin friction pattern at the nose

column. For each row, the angle of attack of the experiment fits to the one of the numerical simulation but a deviation for $\alpha_{\mathrm{AD}}$ exists. The smallest deviation in misalignment between experiment and numerical simulation exists for the first row. Figure 17 shows a remarkable good agreement between the extracted flow structure and the density gradient at the nose and at the wings leading edge. Shock 2 is the reattachment shock of the shear layer in positive $\zeta$-direction*. Shocks 4 and 5 occur due to the misalignment of the AD. The flow physics behind this shock will be given later as well as for shock 6 . Shock 7 does not occur in the pitching plane, it originates from the wing trailing edge. From top to bottom, the deviation in misalignment is increasing. At the nose, due to that fact, the shock structure shows a slight increasing deviation. But at the wings, no worsening occurs. In total, the flow structure that occurs in the experiment is captured very well by the numerical simulation.

Shock 6 in Fig. $17 a$ has its origin in the circumferential circulation of the flow on the missile from windward to lee side. At the lee side itself, the flow direction changes and points along the roll axis, respectively, $x$-coordinate direction (Fig. 18). With increasing $x$-coordinate, more and more fluid approaches from windward and accumulates on lee side. Due to that, the boundary layer thickens on lee side. Finally, the thickening is so large that, the outer supersonic flow is redirected. This leads to shock 6 in Fig. $17 a$.

As has been already mentioned, shocks 4 and 5 occur due to the misalignment of the AD. The effect of the misalignment on the shock structure is sketched in Fig. 19. Without misalignment, the shock angles are identical. More or less symmetric separation bubble occurs and a closed circumferential reattachment

${ }^{*}$ For definition of $\zeta$, see Fig. $20 c$. 


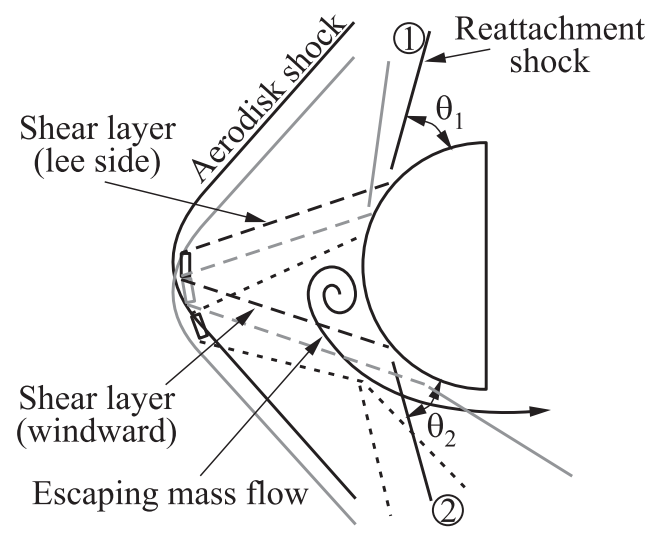

Figure 19 Effect of the misalignment on the shock structure

line exists (see also Figs. $20 a$ and $21 b$ ). Due to the misalignment, the shear layer on windward reattaches more tangential than the shear layer on lee side (see Fig. 19). Hence, a difference in $\theta_{1}$ and $\theta_{2}$ exists. On lee side, a stronger shock occurs with a larger increase in surface pressure at the foot of the shock. An asymmetric but still closed separation bubble develops.

At a certain misalignment, the difference in shock strength respective to pressure increase at the reattachment is so large that the asymmetric separation bubble displaces the shear layer on windward (see green sketch in Fig. 19). The pressure increase on windward that would be at the reattachment due to the shock is not large enough to redirect the oncoming flow of the asymmetric separation bubble. An open reattachment line develops instead of a closed (see Fig. 21c). This leads to an open separation (see Fig. 20b).

The displacement of the shear layer on windward is the reason of shock 4 in Fig. 17a. The coherent structure of the separation bubble dissolves quite fast once it left the separation zone (see Fig. 20b). Hence, the shear layer can reattach and induces shock 5 in Fig. $17 a$.

A further increase in misalignment leads again to a change in flow topology for the separation bubble (see Fig. 20c). The separation bubble induces no displacement of the shear layer on lee side anymore that leads typically to the conical shape of the aerodisk separation. Instead, a straight line connecting the aerodisk and the nose tip is visible for the shear layer on lee side. This can be observed by means of the density gradient in the pitching plane in Fig. $20 c$.

A comparison of the density gradient in the cross sections in Fig. 20 confirms that in case of the largest misalignment, the separation bubble is degenerated. 


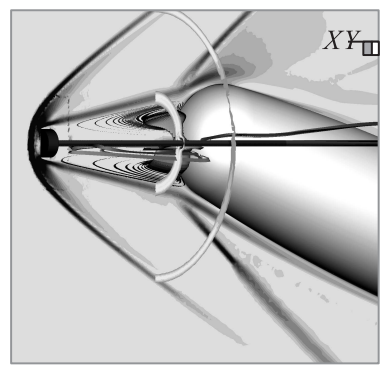

(a)

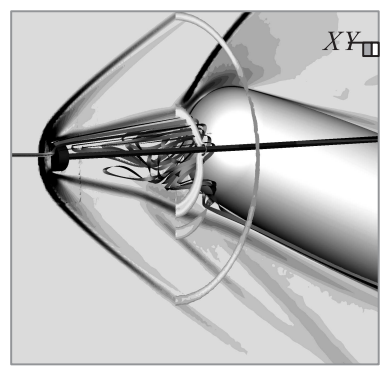

(b)

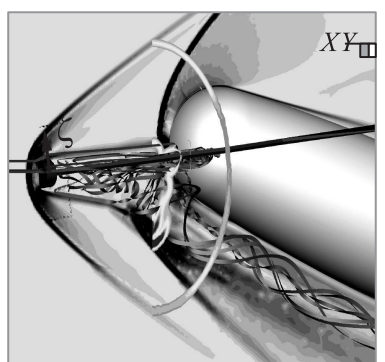

(c)

Figure 20 Comparison of the flow structure and separation bubble at a static angle of attack of $\alpha=21^{\circ}$ for three different misalignments $\left(\alpha=21^{\circ}\right):(a) \alpha_{\mathrm{AD}}=0^{\circ} ;(b) 3^{\circ}$; and $(c) \alpha_{\mathrm{AD}}=7.5^{\circ}$

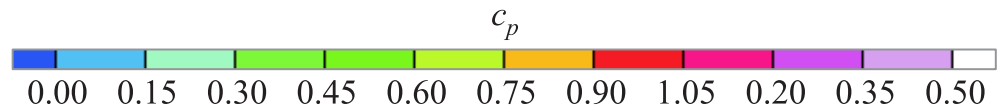

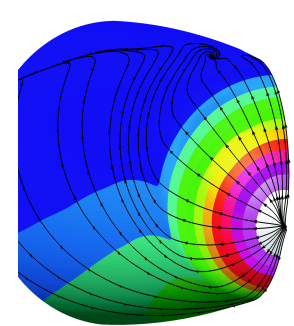

(a)

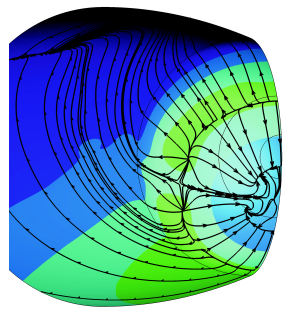

(b)

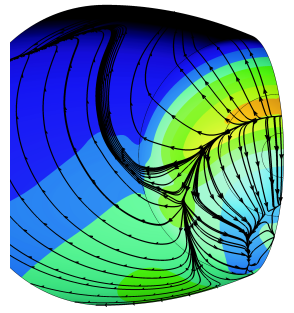

(c)

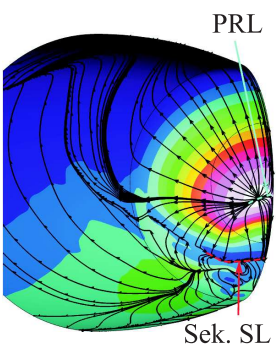

(d)

Figure 21 The $c_{p}$-distributions and skin friction line patterns on the ogive for a static angle of attack of $\alpha=21^{\circ}$ and different misalignments: (a) without $\mathrm{AD} ;(b) \alpha_{\mathrm{AD}}=0^{\circ}$; (c) $3^{\circ} ;$ and $(d) \alpha_{\mathrm{AD}}=7.5^{\circ}$

A vortex develops within the aerodisk separation. In contrast to the vortex in Fig. 20b, it has a longer life time and does not dissolve after it is leaving the separation at the hemispherical nose. The vortex prevents a regular reattachment of the aerodisk shear layer on windward. Hence, shock 5 does not occur for this misalignment.

The change in flow topology with increasing misalignment can be also seen in the skin friction line pattern. A comparison of Figs. $21 c$ and $21 d$ shows that with increasing misalignment, the initial closed reattachment line opens on windward (see Fig. 21c). With a further increase, the reattachment line 
contracts and, finally, a separation line develops pointing in circumferential direction (see Fig. 21d). At this separation line, the flow that reattaches at the reattachment line above separates again and unites with the vortex of the separation.

Figure 21 also illustrates the effect of different misalignments on the $c_{p^{-}}$ distribution at the surface. The corresponding $C_{D}$-values are shown in Table 1.

\section{CONCLUDING REMARKS}

In this paper, a self-aligning aerodisk to reduce the wave drag of a pitching missile was numerically investigated at a Mach number of 2.2. In contrast to a fixed aerodisk, the self-aligning aerodisk is able to pitch about the blunt nose of the missile. With this capability, the aerodisk is able to point into the oncoming flow direction even though the missile itself shows an angle of attack. Due to that also at high angles of attack, a favorable shock system can be induced by the aerodisk. The alignment of the aerodisk is done by aerodynamic forces that are induced at small vanes. To simulate this passive alignment, a coupling of the flow solver with an FMT was necessary. The missile in this investigation is pitching sinusoidal with a frequency of $f=5 \mathrm{~Hz}$ between $\alpha=0^{\circ}$ and $21^{\circ}$ $\left(k_{D}=0.001\right)$. This motion and the Mach number are realistic conditions of an actual missile maneuver. For this test condition, experimental data exist and a comparison can be made with the results of the simulations. Additionally, numerical simulations of a slender body without a self-aligning aerodisk have been performed.

The self-aligning aerodisk successfully reduced the wave drag in the entire range of angle of attack. Although the efficiency decreases with increasing angle of attack at $\alpha=21^{\circ}$, still a drag reduction of at least $5 \%$ remained in comparison to a missile without aerodisk. At $\alpha=0^{\circ}$, the reduction is about $32 \%$. The decrease in efficiency is not due to a failure of the self-alignment rather the decreasing impact of the hemispherical nose on the total drag. Hence, this trend is unpreventable since the aerodisk is just reducing the wave drag at the nose. The alignment works quite well though a misalignment exists that increases with increasing $\alpha$. The maximum in misalignment was about $\alpha_{\mathrm{AD}}=4^{\circ}$. With increasing misalignment, the closed separation downstream of the aerodisk is transformed to an open separation. Although this drastical change in separation occurs, still a reduction of $C_{D}$ is achieved in comparison with a missile without an aerodisk.

A comparison of $C_{D}$ between the simulation and the experiment shows a rather good agreement in experiments and numerical simulation. Larger deviation exists for the drag reduction. This is due to the fact that here, the 
data of the case with aerodisk are processed with the data without an aerodisk. Through this, an adverse accumulation of smaller deviation can have a large impact. But in general, the drag reduction between the experiment and the numerical simulation is of the same order.

As in the numerical simulation, also in the experiment, a misalignment occurs that shows, in general, the same trend and the misalignments are of the same order. Large deviations exist at the reversal points of the motion. This is due to the fact that in the experiment, the center of mass was not located on the pitching axis of the AD. This leads to unwanted hinge moments because of inertial forces. Taking the results of the simulation into account, it is beneficial to locate the center of mass of the $\mathrm{AD}$ at its axis of rotation. A comparison of experimental shadow graphs with numerical density plots in the pitching plane shows a good agreement in the flow structure. The flow solver is capturing well the shocks and the position of them compared to the experiment.

\section{REFERENCES}

1. Eggers, A.J., M. Meyer, D. Resnikoff, and H. Dennis. 1956. Bodies of revolution having minimum drag at high supersonic airspeeds. NACA Technical Report. TN 1306. 555-566.

2. Schülein, E. 2008. Wave drag reduction approach for blunt bodies at high angles of attack: Proof-of-concept experiments. AIAA Paper No. 2008-4000.

3. Alexander, S. R. 1947. Results of tests to determine the effect of a conical windshield on the drag of a bluff body at supersonic speeds. NACA RM. No. L6K08a.

4. Bogdonoff, S. M., and I.E. Vas. 1959. Preliminary investigations of spiked bodies at hypersonic speeds. J. Aerosp. Sci. 26(2):65-74.

5. Chang, P. K. 1970. Separation of flow. New York, NY: Pergamon Press. 777 p.

6. Ahmed, M. Y. M., and N. Qin. 2011. Recent advances in the aerothermodynamics of spiked hypersonic vehicles. Prog. Aerosp. Sci. 47(6):425-449.

7. Tret'yakov, P. K., A. F. Garanin, G. N. Grachev, V. L. Krainev, A. G. Ponomarenko, V. N. Tishchenko, and V. I. Yakolec. 1996. Control of supersonic flow around bodies by means of high-power recurrent optical breakdown. Dokl. Phys. 41(11):566-567.

8. Schülein, E., A. A. Zheltovodov, E. A. Pimonov, and M. S. Loginov. 2010. Experimental and numerical modeling of the bow shock interaction with pulse-heated air bubbles. Int. J. Aerospace Innovations 2(3):183-205.

9. Kim, J.-H., A. Matsuda, T. Sakai, and A. Sasoh. 2011. Wave drag reduction with acting spike induced by laser-pulse energy deposition. AIAA J. 49:2076-2078.

10. Schülein, E., and A. Zheltovodov. 2011. Effects of steady flow heating by arc discharge upstream of non-slender bodies. Shock Waves 21:383-396.

11. Artem'ev, V. I., V. I. Bergel'son, I. V. Nemchinoy, T. I. Orlova, V. A. Smirnov, and V. M. Khazins. 1989. Change of regime in supersonic flow past an obstacle preceded by a thin channel of reduced density. Fluid Dyn. 24(5):779-784. 
12. Meyer, B., H.F. Nelson, and D.W. Riggins. 2001. Hypersonic drag and heattransfer reduction using a forward-facing jet. J. Aircraft 38(4):680-686.

13. Hayashi, K., S. Aso, and Y. Tani. 2005. Numerical study of thermal protection system by opposing jet. 43th AIAA Aerospace Sciences Meeting and Exhibit.

14. Gordeev, V.P., A. V. Krasil'nikov, V. I. Lagutin, and V. N. Otmennikov. 1996. Experimental study of the possibility of reducing supersonic drag by employing plasma technology. Fluid Dyn. 31(2):313-317.

15. Fomin, V.M., A.A. Maslov, N.D. Malmuth, V.P. Fomichev, A.P. Shashkin, T. A. Korotaeva, A. N. Shiplyuk, and G. A. Pozdnyakov. 2002. Influence of a counterflow plasma jet on supersonic blunt-body pressures. AIAA J. 40(6):1170-1177.

16. Shang, J.S. 2002. Plasma injection for hypersonic blunt-body drag reduction. AIAA J. 40(6):1178-1186.

17. Georgievskii, P. Y., and V. A. Levin. 1988. Supersonic flow past bodies in the presence of external heat release sources. Pisma Zh. Tekh. Fiz. 14(8):684-687.

18. Myrabo, L. N., and Yu.P. Raizer. 1994. Laser-induced air spike for advanced transatmospheric vehicles. AIAA Paper No. 1994-2451.

19. Guvernyuk, S. V., and A.B. Samoilov. 1997. Control of supersonic flow around bodies my means of a pulsed heat source. Tech. Phys. Lett. 23:333-336.

20. Knight, D., Y.F. Kolesnichenko, V. Brovkin, and D. Khmara. 2008. High speed flow control using microwave energy deposition. 46th AIAA Aerospace Sciences Meeting and Exhibit.

21. Stalder, J. R., and H. V. Nielsen. 1954. Heat transfer from a hemisphere-cylinder equipped with flow-separation spikes. NACA TN 3287.

22. Gnemmi, P., J. Srulijes, K. Roussel, and K. Runne. 2003. Flowfield around spiketipped bodies for high attack angles at mach 4.5. J. Spacecraft Rockets 40(5):622631.

23. Edney, B. 1968. Anomalous heat transfer and pressure distribution on blunt bodies at hypersonic speeds in the presence of an impinging shock. Flygtekniska Försöksanstalten FFA. Report 115.

24. Schülein, E. 2010. Flying object for transonic or supersonic flight. Patent U.S. $7,7775,480$ B2.

25. Wysocki, O., E. Schülein, and C. Schnepf. 2014. Experimental study on wave drag reduction at slender bodies by a self-aligning aerospike. New results in numerical and experimental fluid mechanics. Springer International Publishing Switzerland. 9:583-590.

26. Schnepf, C., O. Wysocki, and E. Schülein. 2013. Wave drag reduction due to a self-aligning aerodisk. Progress in flight physics. Eds. D. Knight, I. Lipatov, and Ph. Reijasse. EUCASS advances in aerospace sciences book ser. Moscow: TORUS PRESS. 7:475-488.

27. Madrane, A., A. Raichle, and A. Stürmer. 2004. Parallel implementation of a dynamic overset unstructured grid approach. 3rd Conference (International) on Computational Fluid Dynamics Proceeding.

28. Schnepf, C. S., O. C. Wysocki, and E. Schülein. 2014. Numerical investigation on the development of the phantom yaw effect on a maneuvering missile. AIAA Paper No. 2014-3097. 
29. Schwamborn, D., T. Gerhold, and R. Heinrich. 2006. The DLR TAU-code: Recent application in research and industry. European Conference on Computational Fluid Dynamics.

30. DLR. 2012. Technical Documentation of the DLR TAU-code. Release 2012.1.0. 2012.

31. Menter, F. R. 1993. Zonal two equation $k-\omega$ turbulence models for aerodynamic flows. AIAA Paper No. 92-2906.

32. Khalid, M., A. Dujardin, P. Henning, L. Leavit, F. Leopold, M. Mendenhall, S. Prince, and T. Birch. 2004. Application of various turbulence models to investigate the aerodynamic performance of a nasa dual control missile. AIAA Paper No. 2004-5198.

33. Heinrich, R., and A. Michler. 2009. Unsteady simulation of the encounter of a transport aircraft with a generic gust by CFD flight mechanics coupling. CEAS 2009 European Air and Space Conference.

34. Murman, S. M., M. J. Aftosmis, and M. J. Berger. 2003. Simulations of 6-DOF motion with a Cartesian method. AIAA Paper No. 2003-1246. 